\title{
Oncologists' Perceptions of a Digital Tool to Improve Cancer Survivors' Cardiovascular Health
} \author{
Kathryn E. Weaver ${ }^{8}$ \\ 1 Department of Biomedical Informatics, The Ohio State University, \\ College of Medicine, Columbus, Ohio, United States \\ ${ }^{2}$ College of Nursing, The Ohio State University, Columbus, Ohio, \\ United States \\ ${ }^{3}$ Division of General Medicine, Washington University St. Louis, \\ St. Louis, Missouri, United States \\ 4 Institute for Informatics, Institute for Public Health, Washington \\ University, St. Louis, United States \\ ${ }^{5}$ Nationwide Children's Hospital, Columbus, Ohio, United States \\ 6 Ohio State University Wexner Medical Center-Health and \\ Rehabilitation Sciences, Columbus, Ohio, United States \\ ${ }^{7}$ Department of Medical Oncology, The Ohio State University Wexner \\ Medical Center, Columbus, Ohio, United States \\ 8 Wake Forest School of Medicine-Social Sciences and Health Policy, \\ Office of Women in Medicine and Science, Winstom-Salem, North \\ Carolina, United States
}

Marjorie Kelley ${ }^{1,2}$ Randi Foraker ${ }^{3,4}$ En-Ju Deborah Lin ${ }^{5}$ Manjusha Kulkarni ${ }^{6} \quad$ Maryam Lustberg $^{7}$

ACI Open 2019;3:e78-e87.

Address for correspondence Marjorie Kelley, MS, RN, Department of Biomedical Informatics, The Ohio State Univesity, College of Medicine, 1800 Lincoln Tower, Columbus, $\mathrm{OH} 43210$, United States (e-mail: kelley.415@osu.edu).

\section{Abstract}

Keywords

- cancer survivors

- medical informatics

- cardiovascular disease

- lifestyle

- patient participation
Background Cardiovascular (CV) disease continues to be a leading cause of morbidity and mortality with higher rates among cancer survivors than in the general population. Objective This study was aimed to understand oncology providers' attitudes toward a digital CV health tool, delivered via a tablet, to promote CV health in cancer survivors. Methods Using qualitative methods, 14 oncologists, from community and academic practice sites, were interviewed while they used the tool. Interviews were videotaped then analyzed using NVivo 11 software. Themes were inductively developed from the interviews. Results Three major themes emerged from the interviews as follows: (1) system functionality, (2) facilitators and barriers to integration, and (3) appropriate end-users. Oncologists recognized the critical role of $\mathrm{CV}$ health promotion among cancer survivors and identified features about the tool that would be helpful for CV health promotion. Workflow (subtheme) was a barrier to tool use. This feedback enabled tool redesign for further testing in the context of survivorship care.

Conclusion Our findings emphasized the importance of identifying appropriate Endusers which may include other survivorship care providers, patients, and primary care providers.

Implications Our research addresses the knowledge gap in the use of digital tools in cancer survivorship care, specifically digital tools to promote CV health. Future research is needed to evaluate digital tools in cancer survivorship care. Research investigating patients as users of digital tools may provide additional insight. received

June 21,2018

accepted after revision

July 18,2019
DOI https://doi.org/

10.1055/s-0039-1696732.

ISSN 2566-9346. (c) 2019 Georg Thieme Verlag KG

Stuttgart - New York
License terms

(1) (1) 


\section{Background and Significance}

Currently, approximately 15.5 million Americans are cancer survivors, and their prevalence is expected to increase to 19 million by $2024 .{ }^{1}$ Better cancer treatments and earlier detection contribute to the increasing number of survivors. ${ }^{2}$ While more individuals are surviving cancer, their health remains compromised by cardiovascular (CV) disease morbidity and mortality at rates higher than the general population. ${ }^{3-8}$

Causes of CV disease among cancer survivors include the effects of chemotherapy and other anticancer agents, as well as that of radiation..$^{9,10}$ Insulin resistance ${ }^{11,12}$ and higher C-reactive protein levels ${ }^{13}$ are associated with certain cancer treatments. The relatively high prevalence of obesity and physical inactivity during the cancer survivorship period add to the risk of developing CV disease. ${ }^{14}$ Compared with their healthy siblings, cancer survivors are 10 times as likely to develop CV disease. ${ }^{14}$ For all cancer survivors, particularly among those with high long-term survival rates, CV disease is the leading cause of mortality after recurring malignancies. ${ }^{5-8}$

Recent national and international attention underscores the importance of improving health promotion efforts among survivors and cancer survivorship care. ${ }^{15,16}$ The National Cancer Policy Forum of the Institute of Medicine (IOM) and the American Society of Clinical Oncology ${ }^{17-19}$ reiterate the importance of improving cancer survivors' health. Despite a decade of calls for enhanced prevention efforts and care coordination in this population, ${ }^{20-23} \mathrm{CV}$ disease continues to be undertreated. ${ }^{24}$

Digital health technologies have the potential to improve cancer survivorship care ${ }^{25}$ and have demonstrated effectiveness in other settings and populations. ${ }^{26,27}$ The broad category of digital health technology includes such things as mHealth applications designed for smartphones or tablets, electronic health records and patient portals, e-prescribing, wearable devices, telehealth, and telemedicine. ${ }^{28}$ In 2018, the World Health Organization published a taxonomy of digital health interventions under the following four broad categories: (1) clients, (2) health care providers, (3) health system managers, and (4) data services. ${ }^{29}$ Health care providers use digital technologies to improve access, reduce costs, improve efficiency and quality, and make care more personalized in addition to offering care that transcends time and distance. Patients can track and manage their health and wellness and become more engaged in their care. Digital health technologies or digital tools used in the context of this study are defined as technology, such as mobile applications, delivered via tablet or smartphone, or integrated within an electronic health record (EHR) system to increase information exchange, improve survivorprovider connection and communication, and to delivery of health services.

Currently, available digital tools have increased cessation counseling for smokers, ${ }^{30}$ facilitated goal-setting in prediabetic patients, ${ }^{31}$ improved dyslipidemia treatment, ${ }^{32}$ and medication management. ${ }^{33}$ Superior patient outcomes and cost savings have been demonstrated by the use of such tools in cardiothoracic surgery, ${ }^{34}$ HIV care, ${ }^{35}$ CV health, ${ }^{26,27}$ and dyslipidemia treatment. $^{32}$
Few digital tools have been rigorously evaluated in cancer survivorship care. ${ }^{36}$ A metareview of the effects of technologies on patients with cancer identified positive effects on patient knowledge, information, and support. Findings for psychological and physical well-being, as well as the quality of life, were inconsistent or lacking. ${ }^{36}$ Berry and colleagues ${ }^{37}$ conducted one of a limited number of randomized control trials investigating a web-based intervention on symptom distress during cancer therapy. Web-based self-care support and communication coaching, as well as symptom and quality of life screening, reduced symptom distress. ${ }^{37}$ In a pilot study, Jaatun and colleagues $^{38}$ investigated the feasibility of using a digital visualization tool in the management of advanced cancer-related pain. Patients and providers agreed that the tool had the potential to improve pain management and communication.

However, the tool was not evaluated for efficacy. ${ }^{38} \mathrm{Re}-$ search investigating digital tools focused on lifestyle behavior change among cancer survivors offers mixed evidence of effect for physical activity and diet on health outcomes and is limited by lack of scientific rigor. ${ }^{39}$ None of the technologies to date have been integrated into the EHR. To the authors' knowledge, no digital tools have been designed specifically to address CV health promotion and disease prevention in survivorship care. A gap exists in the scientific knowledge base underpinning the development and research of such digital tools. However, before such promising tools can be developed for survivorship care, barriers and enablers of use, specific to survivorship care providers, must be examined. Good digital intervention design requires a clear understanding of users' needs. ${ }^{40,41}$

\section{Objective}

Therefore, the objective of this qualitative study was to understand oncology providers' needs and attitudes toward an EHR-based tool, originally designed to promote the CV health of patients in the primary care setting. Two aims supported this objective are (1) assessment of the usability of the CV health tool as part of cancer survivorship care within the oncology setting and (2) identification of enablers and barriers to tool use in the oncology setting.

\section{Methods}

\section{Design}

A qualitative design using a semistructured interview technique enabled data collection from oncology physicians. Due to the exploratory nature of the research questions, a qualitative method of inquiry was selected to achieve more complete insights into providers' expectations of technology use for improving CV health in survivorship care. The technology, a CV health promotion tool, ${ }^{26}$ was designed to be shared by health care providers with patients at the point of care. Detailed description and evaluation of the tool in the primary care setting have been previously published. ${ }^{26,27,42}$ Briefly, a link to the tool was available to primary care providers within the EHR. The tool can be used on any given EHR platform. Patient information from the EHR populated 
the tool fields (i.e., patient weight and blood pressure) and was used to calculate the patient's "CV health score." The CV health score is a health metric defined by the American Heart Association by the presence of seven health behaviors and health factors (i.e., nonsmoking and physical activity). ${ }^{43}$ The tool was shared with the patient and provider via the EHR computer screen offering a starting point for discussions about CV health. The providers used the interactive features to demonstrate how changes in each CV health component (i.e., decreasing weight and stop smoking) could improve overall CV health.

Observation, interviewing, and content analysis are commonly-used techniques to help researchers to understand stakeholders' needs during all phases of design, development, and evaluation of technology tools, including the use of health informatics tools. ${ }^{44}$ Oncology providers' needs may be different than those of primary care providers. To understand the differences in the context of the CV tool used in oncology versus primary care, we chose qualitative methods to better inform tool redesign. These qualitative techniques have been used in the development of clinical decision-support systems, ${ }^{45}$ shared decision-making tools, ${ }^{46}$ patient portal tools, ${ }^{47}$ as well as in other health information technology applications. ${ }^{48,49}$

\section{Setting/Participants}

One academic and one community oncology practice in North Carolina were selected as recruitment sites. Both sites treat patients enrolled in the National Cancer Institute's Network of Community Oncology Research Program (NCORP) practices. The study was jointly approved by the Institutional Review Boards at Wake Forest University and the Ohio State University.

Oncologists, caring for patients with longer expected survival times postdiagnosis, were recruited. Oncologists met the following inclusion criteria: 18 years of age or older, actively practicing in patient care as a board-certified oncologist in the United States, and able to speak and read English. After providing informed consent, enrollment occurred regardless of race, gender, age, or ethnicity. The oncologists received a $\$ 25$ gift card for their participation.

\section{Procedures}

Inperson semistructured interviews were conducted and video recorded by study-to-study investigators. Interview prompts can be found in -Supplementary Appendix A (available in the online version). Video recordings captured verbal answers and use of the CV health tool, a software application presented on a tablet ( - Fig. 1). Video recordings were used to capture nonverbal communication, voice inflection, and emotion, thus adding to our ability to triangulate data. All study materials were deidentified, and the audio portions of the recordings were transcribed verbatim then checked for accuracy by two authors.

Data were organized and analyzed using NVivo 11 (QSR International Pty. Ltd., 2015) software. Transcripts and demographic data were imported into the software along with field notes with descriptions and nonverbal responses (i.e., hand gestures) which provided context to the transcripts.

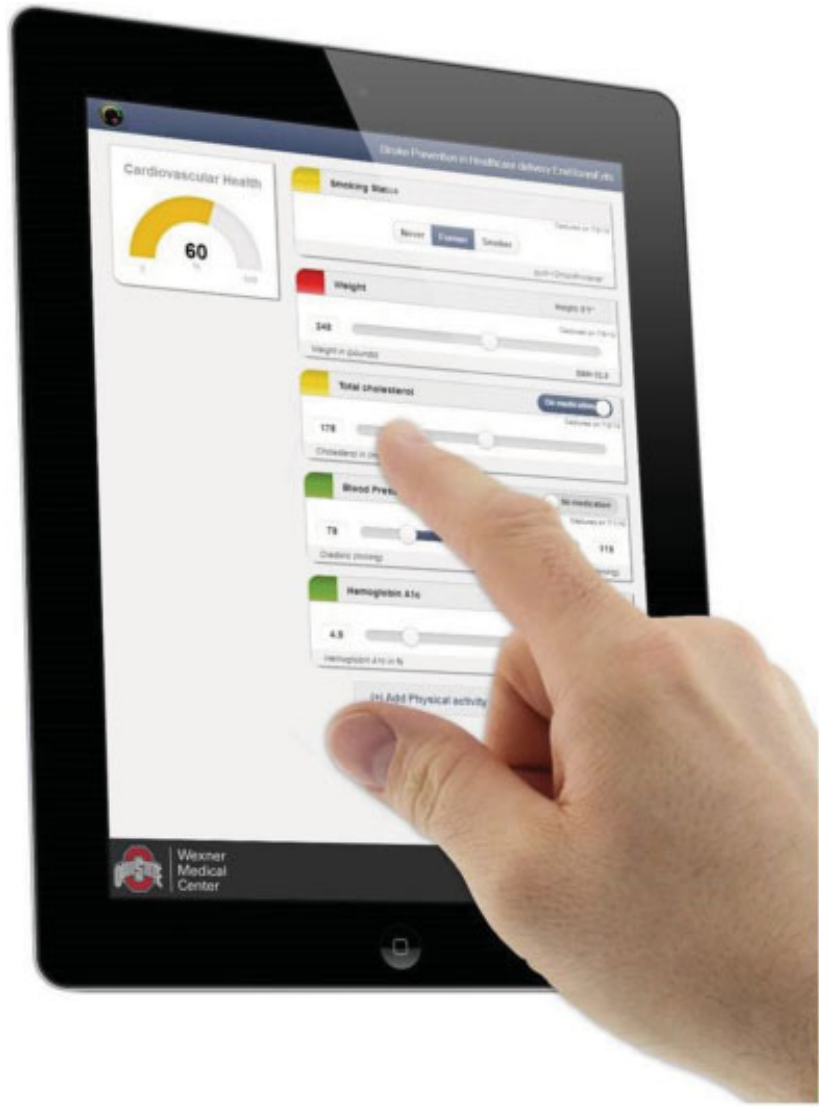

Fig. 1 Study software application presented on a tablet.

\section{Results}

Fourteen oncologists, 50\% female, were interviewed for our study ( - Table 1). All oncologists completed all aspects of the study and generated 14 video files, ranging in length from approximately 10 to 30 minutes. Most participants reported spending greater than $75 \%$ of their time in direct patient care $(n=11)$, with half working in oncology practice less than 11 years. Most providers (11/14) thought it was important to discuss CV health behaviors with survivors; however, only three reported that they "usually" took time to have CV health discussions with their patients.

The data were coded using guidelines developed by Sandelowski ${ }^{50}$ for inductive content analysis. Two authors highlighted, extracted, and condensed text in an inductive process to identify concepts that might develop into themes. Weekly discussions and iterative coding, over 2 months, helped to develop the initial coding schema. Concurrently, the Code Book ( - Supplementary Appendix B, available in the online version) and coding definitions were created. This iterative process continued until thematic saturation was reached.

Several techniques helped to ensure the qualitative study rigor. ${ }^{51,52}$ We employed step-by-step comparisons of independent investigator coding. Qualitative evidence (participant quotes) used in the development of the Code Book helped to increase study transparency, coding accuracy, and trustworthiness as did inclusion and exclusion criteria 
Table 1 Demographics of oncologist participants $(n=14)$

\begin{tabular}{|l|l|}
\hline Variables & Number of participants (\%) \\
\hline Gender & $7(50)$ \\
\hline Female & $7(50)$ \\
\hline Male & \\
\hline Practice site & $4(28.6)$ \\
\hline Academic & $10(71.4)$ \\
\hline Community & \\
\hline $\begin{array}{l}\text { Oncology } \\
\text { practice type }\end{array}$ & $11(78.6)$ \\
\hline Hematology or medical & $2(14.3)$ \\
\hline Gynecological & $1(7.1)$ \\
\hline Radiation & \\
\hline Years in practice & $2(14.3)$ \\
\hline$<1$ & $1(7.1)$ \\
\hline $1-5$ & $4(28.6)$ \\
\hline $6-10$ & $1(7.1)$ \\
\hline $11-20$ & $6(42.9)$ \\
\hline$>20$ & \\
\hline $\begin{array}{l}\text { Time spent in direct } \\
\text { patient care (\%) }\end{array}$ & $2(14.3)$ \\
\hline $25-50$ & $1(7.1)$ \\
\hline $51-75$ & $11(78.6)$ \\
\hline$>75$ & \\
\hline
\end{tabular}

associated with each theme. Peer review and debriefing of the Code Book for theme completeness and discreteness enhanced credibility and confirmability of the themes through external validation. Peer reviewers included a medical oncologist outside the North Carolina area, a nurse informatician $(\mathrm{PhD})$ with experience in health care informatics usability testing, and a computer scientist (PhD) working in health informatics. Detailed decision logs with decision justifications, reflective memoing, and a date/time stamped audit trail also enhanced study rigor.

To ensure intercoder reliability, three coders independently coded 29\% (4 of 14) of the transcripts. Differences in coding were resolved through discussion. To ensure intracoder reliability, one investigator coded of all the audio recordings and then recoded $43 \%$ of the transcripts ( 6 of 14 ) a second time. Only a total of six discrepancies were noted and resolved through discussion with other coders. Themes were exhaustive and mutually exclusive, thus ensuring close representation of the text and avoiding ambiguity. ${ }^{53}$

Finally, these data were presented to a clinical advisory group comprised of an oncologist, a cardiologist, and an oncocardiologists to determine how to refine the existing CV health tool, in the best possible way, to meet the needs of providers in the cancer survivorship care setting.

Three major themes emerged from the data ( - Table 2 ) are (1) system functionality, (2) facilitators and barriers to
Table 2 Themes and subthemes identified in transcript review

\begin{tabular}{|l|l|l|}
\hline Themes & Subthemes & $\begin{array}{l}\text { Subordinate to } \\
\text { subthemes }\end{array}$ \\
\hline $\begin{array}{l}\text { System } \\
\text { functionality }\end{array}$ & & \\
\hline & $\begin{array}{l}\text { Architecture } \\
\text { functionality }\end{array}$ & \\
\hline & $\begin{array}{l}\text { Interface } \\
\text { functionality }\end{array}$ & \\
\hline & content & \\
\hline & & Physical activity \\
\hline & & Diet \\
\hline & Objective clinical data \\
\hline & CV health score & Other \\
\hline $\begin{array}{l}\text { Appropriate } \\
\text { end-users }\end{array}$ & & \\
\hline & $\begin{array}{l}\text { Oncologists } \\
\text { as users }\end{array}$ & \\
\hline & Others as users & \\
\hline $\begin{array}{l}\text { Facilitators } \\
\text { and barriers } \\
\text { to integration }\end{array}$ & & \\
\hline & Use & \\
\hline & Workflow & \\
\hline & $\begin{array}{l}\text { Contextual } \\
\text { influences }\end{array}$ & \\
\hline & & \\
\hline
\end{tabular}

integration, and (3) appropriate end-users. The system functionality theme contained the most subthemes, with the subtheme of interface functionality discussed most frequently and by all fourteen oncologists. Additionally, most oncologists (12/14) made comments about appropriate endusers and workflow. A complete listing of operational definitions, exemplars, and inclusion/exclusion criteria for each theme can be found in the Code Book (- Supplementary Appendix B). Barriers and enablers according to each theme and subtheme are described in more detail in the sections that follow. Briefly, most oncologists discussed ideas about different users, made suggestions for future interface functionality, and had questions about the CV health score. Most oncologists were also concerned about their workflow while using the tool.

\section{System Functionality (Theme 1)}

The major theme of system functionality includes the following interdependent parts of the health information technology system: hardware, software, user interface, and information content. For these semistructured interviews, this theme contained many subthemes including architectural functionality, interface functionality, content, and CV health score ( - Table 2). The subtheme content was further divided into diet, physical activity, objective data, and other with 10 providers offering thoughts about the clinical content included 
in the tool. Most content comments consisted of suggestions for additional content for a specific cancer patient population, with many centering on their desire to see the inclusion of cancer treatment data in the tool.

The most prevalent subtheme, interface functionality, referred to the human-computer interaction or the users' perception of the functions of the interface. Interface functionality includes all aspects of the system which is seen, touched, or heard. ${ }^{54}$ For our study, we included comments about ease of use, the sensitivity of the buttons, and precision of the slider bars to touch. Comments concerning content or hardware interoperability (printing screen) were excluded from this subtheme. While all oncologists made comments about the interface functionality, their reviews were mixed. For 12 providers, interface functionality acted as an enabler of use. One oncologist stated as follows:

"It's visually friendly and interactive. I like that and very user-friendly."

Conversely, 10 providers identified interface functionality features, as barriers to use, with several commenting on the sensitivity of the slider bars when the tool was presented on a tablet device for the interviews. Interestingly, most enabling statements (63\%) concerning interface functionality emanated from oncologists with less than 11 years of experience in oncology. This was the same group with the most interactions per minute with the CV health tool.

Nonverbal interaction patterns with the CV health tool differed based upon the length of the oncologists' practice experience. Oncologists with less than 11 years of practice experience $(n=7)$, clicked and interacted with the tool at a mean rate of 16.1 interactions/minute (standard deviation $[S D]=7.8)$. In contrast, oncologists in practice longer $(\geq 11$ years) clicked and interacted with the tool at a mean rate of 4.1 interactions/minute $(\mathrm{SD}=3.4)$.

The subtheme CV health score received comments from all oncologists. This score, presented on a continuum of poor $(0)$ to ideal (100) CV health, comprises seven modifiable items (behaviors and factors) affecting the CV health of individuals. The seven items include smoking, body mass index, physical activity, diet, total cholesterol, blood pressure, and blood glucose. ${ }^{26,27,43,55}$ The CV health score reflects an American Heart Association practice initiative. ${ }^{43,55}$ Several providers indicated that health behaviors, such as smoking, body mass index, physical activity, and diet were relevant to their practice but total cholesterol, blood pressure, and blood glucose were typically not in their purview.

\section{Appropriate End-Users (Theme 2)}

The next most prevalent theme encompassed comments about appropriate end-users. We defined appropriate endusers as "individuals using and involved in all aspects of the design, redesign, development, implementation, security, and use of the health information technology (HIT). ${ }^{56 "}$ In our definition, we included all types of providers, as well as patients, caregivers, and family members. ${ }^{57}$ Twelve of the 14 oncologists made comments about who should be the user of this CV health tool as it relates to survivorship care, with all oncologists suggesting additional appropriate end-users.
One oncologist, who routinely initiated conversations with patients about CV health, stated as follows:

“... their initial appointment with us ... . I think (this CV health tool) would be very helpful then."

This same oncologist felt the application would be useful for shared decision making:

"I think this would be a good tool to get in and show something visual (to patients) so that we can kind of work through together."

Twelve oncologists felt that CV health was important to address during survivorship care and suggested others as users, primary care practitioners, advanced practice nurses, and the patients themselves. Many made statements such as:

“... you go to a primary care practitioner because you are worried about your health. ... oncologists are not particularly worried about (CV health)."

The subthemes of workflow and use elucidated reasons for the shift in survivorship care to primary care practitioners and are included in the next theme.

\section{Facilitators and Barriers to Integration (Theme 3)}

We defined integration in terms of use, "the ability of the system to achieve a goal." ${ }^{41}$ However, an important corollary for our theme was the "capability of the software product to enable specified users to achieve specified goals with effectiveness, productivity, safety, and satisfaction in specific contexts of use. $^{58 "}$ In other words, whether the tool could be integrated into clinical practice and what were the facilitators and barriers in doing so. With this corollary in mind, three subthemes emerged, use, workflow, and contextual influences. The most prevalent comments from the oncologists' concern were workflow, with 12 of 14 oncologists identified lack of time as the reason why health promotion discussions are difficult to include in the workflow. One summed up this sentiment by saying,

"I would not like this application if it would take more than a couple of minutes during the clinic time .... If going through this generates a lot of nononcological time, then I wouldn't like using it."

In summary, oncologists realized the need for survivorship care, including particular attention to the CV health of their patients, but time and workflow were significant barriers.

Overall, the appropriateness of oncologists as end-users, problems incorporating such a tool into oncologists' workflow, and nononcology time to counsel patients on CV health behavior change were cited as the largest barriers to tool use by the oncologists. Notably, most oncologists questioned the meaning of the CV health score metric as they were unfamiliar with the American Heart Association metric of modifiable factors and behaviors to improve CV health. The oncologists offered substantive suggestions for tool redesign, such as 
adding links to information about the CV health and adding age-adjusted laboratory norms for quick reference for providers and for personalizing the patient experience, visualization of patient progress over time, personalized to that patient's current level of physical activity, or perhaps radiation dose, and chemotherapies received. Oncologists also suggested customization of the tool for specific cancer types (i.e., breast and prostate).

As a result of the above findings and following consultation with the clinical advisory group, the CV health tool was refined for use in cancer survivorship care (-Fig. 2). Specifically, a tab was added which contained data on potentially cardiotoxic treatments received, and CV health behaviors were moved to the top of the CV health tab, while CV health factors were transitioned to the bottom. However, further research is necessary to understand the usefulness of these and other suggested features, as well as the usefulness of the tool, by other provider groups (i.e., nurses, dieticians, physical therapists, and primary care practitioners).
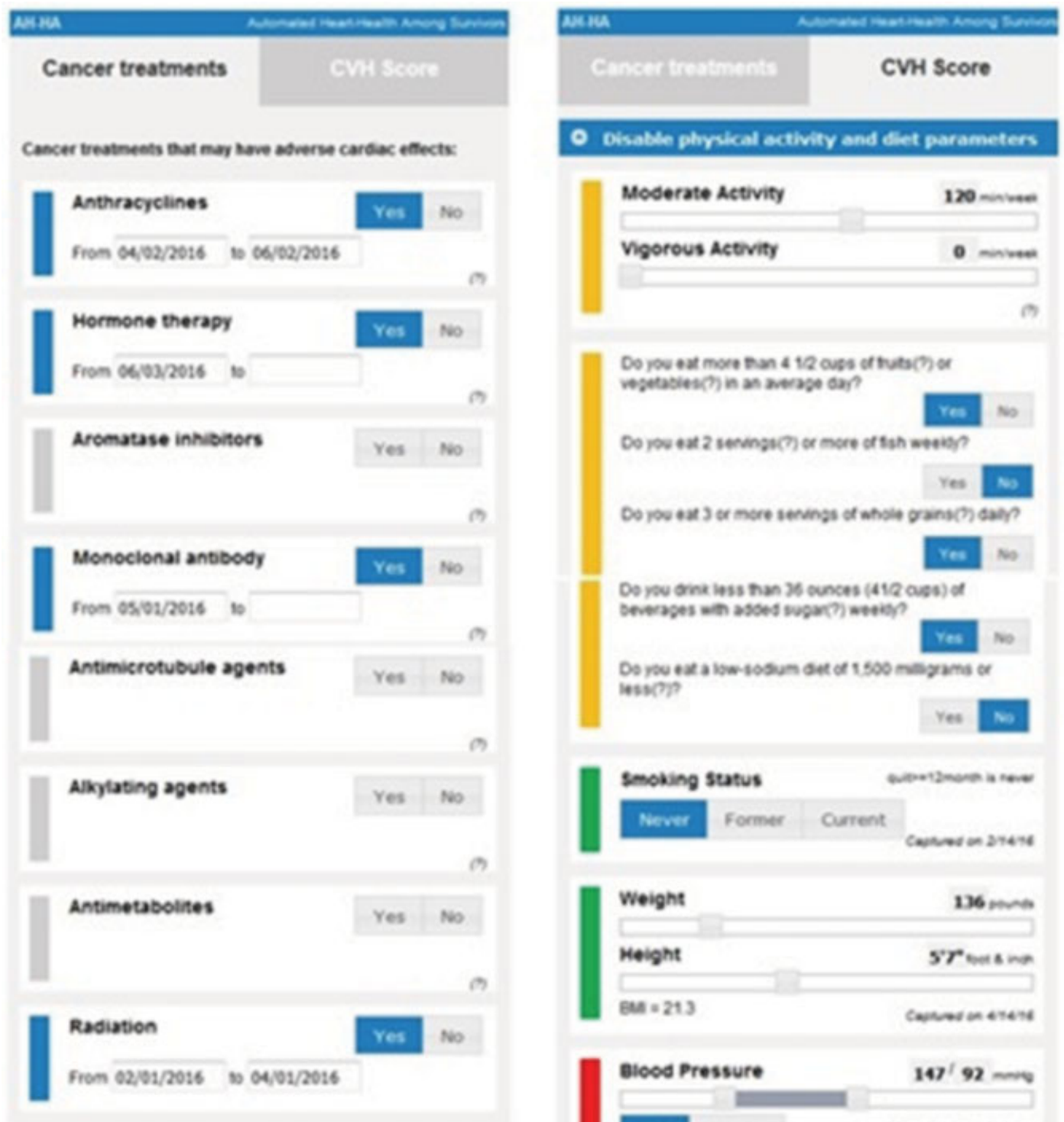

Do pow eat more han 412 oves ut huter or

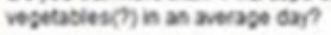

Do pos ext 2 seninguch or more of tah wevee?

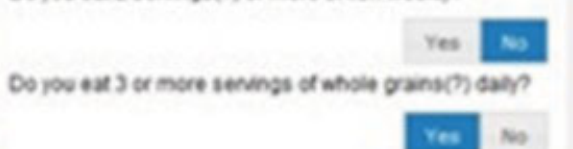

Do you aink iess han 36 ounces (412 ouss) of bevages wh asces wogr? wetion?
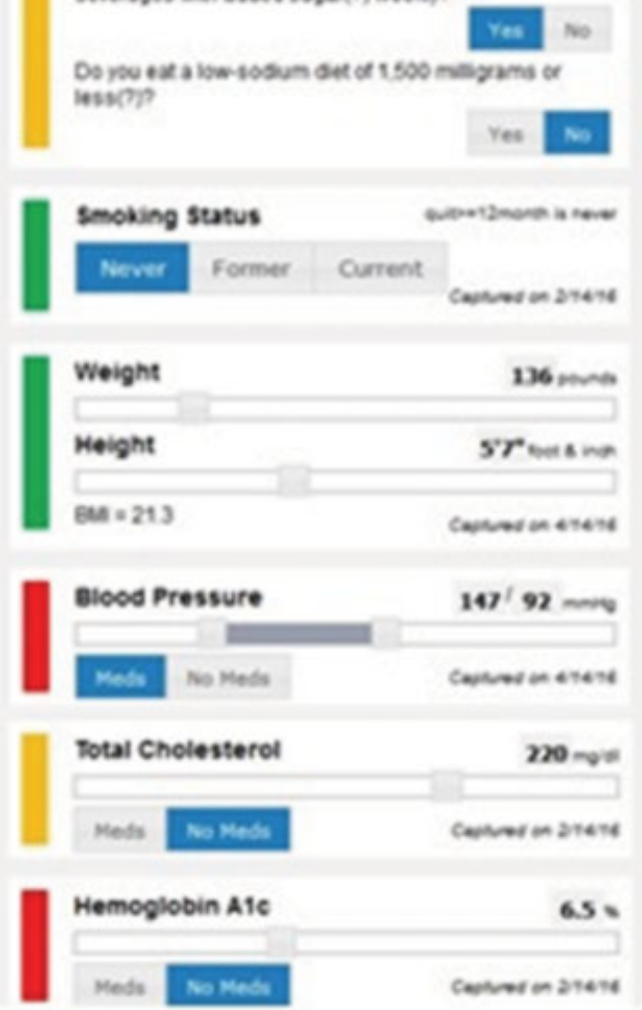

Fig. 2 Screenshot of adapted CV health tool. CV, cardiovascular. 


\section{Discussion}

To our knowledge, this qualitative study is the first to examine the usability of a CV health tool, linked to an EHR, in cancer care and the first to evaluate oncologists' perception of a digital tool. Use of a CV health tool by oncologists to support survivorship care represents a complex intersection between the themes of system functionality, appropriate end-users, and facilitators and barriers to integration. We identified enablers and barriers depending upon the specific feature or context of tool use. Importantly, the most significant findings of our study centered on appropriate end-users and the importance of engaging others (primary care practitioners, nurses, and patients themselves) as the appropriate end-users. West and colleagues $^{59}$ reported primary care physicians and nurses as being more flexible in using tools based on heterogeneous and varied information sources, while secondary care specialists, often expressed the need for standardized information before being able to evaluate it effectively. While oncologists in our study failed to remark on the need for standardization, they did identify other practitioners as appropriate end-users of the CV health tool. However, most of the appropriate endusers' thematic content in this study was a function of the need for primary care practitioners to manage $\mathrm{CV}$ risk in survivorship care and was not necessarily a function of the technology itself. This recurring discordance among care practitioners is not new and continues to cause deficiencies in care. ${ }^{60-62}$ For example, recent systematic literature reviews, involving patients' and primary care providers' perspectives of cancersurvivorship care, identified the need for survivorship care in the primary health care sector. ${ }^{63,64}$

Major enablers of use of the CV health tool included the user-centered design of the interface and the potential to engage patients. Oncologists supported the importance of patient engagement in CV health and thought the tool could engage both providers and patients due to the visual appeal. Of note, the enabling features of interface functionality and the overall visual user experience of the tool were many, color scheme, ease of use, and interactive parameters which demonstrate the impact of behavior change on $\mathrm{CV}$ health. The importance of this type of data synthesis mirrors work by Berry and colleagues, while investigating clinicians' perspectives of an e-health tool addressing symptoms and quality of life during cancer therapy. ${ }^{65}$ The importance of interface functionality identified by the oncologists in this study, including ease of use and user-centered tailoring, is supported by the work of Puszkiewicz and colleagues ${ }^{66}$ and Robertson and colleagues. ${ }^{67}$ In both studies, researchers identified the importance of goals coming from a trusted source and the importance of data visualization. While the participants in both studies were cancer survivors, they did echo the oncologists' beliefs about interface functionality and patient engagement. Because our CV health tool individualized all patient data for each provider-patient meeting, oncologists recognized the engagement power of such personalized tailoring. The tool offered immediate feedback on current CV health status, as well as evidenced-based goals, to attain better CV health.
Puszkiewicz et al also reported the utility of digital tools for providers ${ }^{66}$ regarding time demands, a theme discussed by our oncologists. Easy to use tools, offer a quick opportunity to discuss CV health with patients in a time demanding environment. Oncologist-patient discussions around lifestyle behaviors are critical for oncologists treating teenage and young adult cancer survivors as oncologists are the primary source of information on lifestyle behavior for these survivors. ${ }^{68}$ Our findings are also consistent with results reported in recent pilot studies investigating digital tools to improve lifestyle behaviors in cancer survivors, specifically the importance of ease of use, and convenience for patients and providers. ${ }^{69,70}$ We recognize, however, that certain subsets of survivors are at greater risk of cardiotoxicity and that the use of a digital tool to discuss lifestyle modification should not displace referrals to specialists in oncocardiology in such cases. For example, survivors receiving anthracycline based chemotherapy, ${ }^{71}$ receiving immunotherapy, or those with a positive diagnoses for human epidermal growth factor receptor 2 (HER2) and receiving targeted therapies for HER $2,{ }^{72}$ as well as survivors with left breast radiation or other radiation near the heart ${ }^{73}$ may benefit from referral to an oncocardiologist. Until the development of evidence-based guidelines for $\mathrm{CV}$ risk assessment (before and after cancer therapy), ${ }^{74,75}$ providers should err on the side of caution particularly with those patients most at risk for cardiotoxicity.

Findings and themes identified in this research support those described by others conducting qualitative work in noncancer health care technology. For example, our work mirrored seven of the eight themes described in the Sociotechnical Model as follows: (1) hardware and software computing infrastructure, (2) clinical content, (3) humancomputer interface, (4) people, (5) workflow and communication, (6) internal organizational policies, procedures, and culture, and (7) external rules, regulations and pressures. ${ }^{54}$ These themes are interconnected and often dependent on one another in terms of change and effect. Similarly, our user (oncologist) expectancies of the CV health tool were influenced by workflow, infrastructure, and human-computer interactions. Our findings also parallel those reported over decades by others involved in health information technology design and implementation. ${ }^{76-78}$ Recently, West and colleagues ${ }^{59}$ described variations in data visualization, data granularity, and units of measure acted as barriers to use as did lack of contextual information. ${ }^{59}$ These findings supported our work in that oncologists identified good data visualization as an enabler but made suggestions to include other patient information (granularity) to provide context (i.e., trends over time).

The oncologists interviewed offered substantive suggestions for tool redesign which were translated into the refined tool. While we purposively sampled oncologists from both academic and community oncology practices in North Carolina, our findings cannot be generalized beyond such practices. We failed to include other providers in our sample. Nurses, nurse practitioners, dieticians, exercise physiologists, and physical therapists, as well as behavior change specialists, may have offered different insights and would have broadened the perspectives gained. The patients' perspectives were also 
missing from this qualitative study. Our interviews were conducted, while we were asking oncologists to use and interact with the digital tool. Multitasking may have limited oncologists' ability to fully communicate their thoughts and ideas. In addition, interviews were brief. As learnability and use experience are moderators of usability, oncologists may not have had enough time to learn how to use the tool to formulate informed perceptions. In addition, age is a known moderator of technology use. The age of oncologists was not assessed; only years of experience in oncology practice. In a study conducted in Germany, breast cancer physicians up to age 60 had high rates of acceptance for internet supported tools, while those above the age of 60 exhibited a decrease in acceptance levels. ${ }^{79}$ However, rates never dropped below $50 \%$ in any age bracket. Of physicians favoring smartphone patient support, the highest percentages (90.5\%) of acceptance were among providers 30 to 39 years of age. ${ }^{79}$ Using years of practice as a proxy for age, our results confirm these findings.

\section{Conclusion}

This original study identified enablers and barriers to use of a CV health tool, by oncologists, in the cancer survivorship care setting. Identifying stakeholders' needs, including barriers and enablers of use, are necessary for good digital design at every stage of the development lifecycle. ${ }^{40,41}$ Oncologists supported the use of the tool in cancer-survivorship care and agreed that a visual, interactive tool could improve patient engagement and education; both of which appear as critical to positive patient outcomes during cancer-survivorship care. However, oncologists' time demands in the clinic dampened oncologists' enthusiasm for the use of the CV health tool Oncologists thought other health care providers (i.e., nurses, nurse practitioners, dieticians, and physical therapists) might be better suited to support survivors' health-behavior change using the tool.

Additionally, the ongoing discordance between oncologists and primary care providers over survivorship care acted as a barrier to use. Oncologists understood the importance of using digital tools to help improve the CV health of cancer survivors but felt engagement of primary care providers, other health care providers (i.e., nurse practitioners and nurses), and patients were central to its success. Future research is needed to understand the perspectives of oncologists and cancer survivors regarding the refined CV health tool. This research may help to inform the design, development, and continued rigorous study of other digital tools to improve survivorship care and ultimately improve the health of patients surviving cancer.

\section{Clinical Relevance Statement}

For over a decade, the American Society of Clinical Oncology and the National Cancer Policy Forum of the Institute of Medicine have called for the improvement of health promotion efforts for cancer survivors. Current survivor clinical guidelines outline the importance of diet and exercise in the survivorship period. However, cardiovascular disease in this population continues to be undertreated. This research begins to address the scientific knowledge gap in using novel digital tools to address the problem of cardiovascular disease in patients who survived cancer.

\section{Protection of Human and Animal Subjects}

This study was performed in compliance with the ethical standards of the World Medical Association Declaration of Helsinki on Ethical Principles for Medical Research Involving Human Subjects and was reviewed and approved by the Institutional Review Boards of Wake Forest University and the Ohio State University.

Note

The content is solely the responsibility of the authors and does not necessarily represent the official views of the NIH or the NLM.

\section{Funding}

This project was supported by the National Center for Advancing Translational Sciences (NCATS), National Institutes of Health, through Grant Award Number UL1TR002733. This work was partially supported by a grant from the National Institute of Health, NCI R01 CA226078.

\section{Conflict of Interest}

None declared.

\section{References}

1 American Cancer Society. Cancer Facts \& Figures 2019. Available at: http://www.cancer.org/research/cancer-facts-statistics/all-cancerfacts-figures/cancer-facts-figures-2019. Accessed September 2019

2 de Moor JS, Mariotto AB, Parry C, et al. Cancer survivors in the United States: prevalence across the survivorship trajectory and implications for care. Cancer Epidemiol Biomarkers Prev 2013;22 (04):561-570

3 Fung C, Fossa SD, Milano MT, Sahasrabudhe DM, Peterson DR, Travis LB. Cardiovascular disease mortality after chemotherapy or surgery for testicular nonseminoma: a population-based study. JClin Oncol 2015;33(28):3105-3115

4 Youn P, Milano MT, Constine LS, Travis LB. Long-term causespecific mortality in survivors of adolescent and young adult bone and soft tissue sarcoma: a population-based study of 28,844 patients. Cancer 2014;120(15):2334-2342

5 Daher IN, Daigle TR, Bhatia N, Durand JB. The prevention of cardiovascular disease in cancer survivors. Tex Heart Inst J 2012;39(02):190-198

6 Lipshultz SE, Adams MJ, Colan SD, et al; American Heart Association Congenital Heart Defects Committee of the Council on Cardiovascular Disease in the Young, Council on Basic Cardiovascular Sciences, Council on Cardiovascular and Stroke Nursing, Council on Cardiovascular Radiolo. Long-term cardiovascular toxicity in children, adolescents, and young adults who receive cancer therapy: pathophysiology, course, monitoring, management, prevention, and research directions: a scientific statement from the American Heart Association. Circulation 2013;128(17): 1927-1995

7 Ward KK, Shah NR, Saenz CC, McHale MT, Alvarez EA, Plaxe SC. Cardiovascular disease is the leading cause of death among endometrial cancer patients. Gynecol Oncol 2012;126(02): 176-179 
8 Weaver KE, Foraker RE, Alfano CM, et al. Cardiovascular risk factors among long-term survivors of breast, prostate, colorectal, and gynecologic cancers: a gap in survivorship care? JCancer Surviv 2013;7(02):253-261

9 Adams MJ, Lipshultz SE. Pathophysiology of anthracycline- and radiation-associated cardiomyopathies: implications for screening and prevention. Pediatr Blood Cancer 2005;44(07):600-606

10 Mertens AC, Liu Q Neglia JP, et al. Cause-specific late mortality among 5-year survivors of childhood cancer: the Childhood Cancer Survivor Study. JNatl Cancer Inst 2008;100(19): 1368-1379

11 Coughlin SS, Smith SA. The insulin-like growth factor axis, adipokines, physical activity, and obesity in relation to breast cancer incidence and recurrence. Cancer Clin Oncol 2015;4(02): 24-31

12 Greenfield DM, Blewitt A, Coleman RE, et al. Evaluation of adipocytokines and traditional cardiometabolic risk factors in young male cancer survivors: an age-matched control study. Clin Endocrinol (Oxf) 2016;84(02):296-304

13 Wethal T, Haugnes HS, Kjekshus J, et al. C-reactive protein; a potential marker of second cancer and cardiovascular disease in testicular cancer survivors? Eur J Cancer 2010;46(18):3425-3433

14 Oeffinger KC, Mertens AC, Sklar CA, et al; Childhood Cancer Survivor Study. Chronic health conditions in adult survivors of childhood cancer. N Engl J Med 2006;355(15):1572-1582

15 Pavon JM, Zhao Y, McConnell E, Hastings SN. Identifying risk of readmission in hospitalized elderly adults through inpatient medication exposure. J Am Geriatr Soc 2014;62(06):1116-1121

16 van Dorp W, Mulder RL, Kremer LC, et al. Recommendations for premature ovarian insufficiency surveillance for female survivors of childhood, adolescent, and young adult cancer: A report from the international late effects of childhood cancer guideline harmonization group in collaboration with the pancaresurfup consortium. JClin Oncol 2016;34(28):3440-3450

17 ASCO. Cancer survivorship. 2015; Available at: https://www. cancer.net/survivorship. Accessed September 10, 2015

18 The National Academics of Science, Engineering, Medicine: health and medicine Division.. National cancer policy forum. 2013; Available at: http://iom.nationalacademies.org/activities/disease/ncpf/2013jul-15/day\%202/session\%205/25-freyer.aspx. Accessed October 1, 2015

19 Hewitt M, Ganz PA. A National Coalition for Cancer Survivorship and Institute of Medicine National Cancer Policy Forum Workshop. 2006. Available at: http://www.nap.edu/read//11739/chapter/1. Accessed September 2019

20 Institute of Medicine. From cancer patient to cancer survivor: Lost in Transition. 2005. Available at: http://www.nationalacademies.org/hmd/Reports/2005/From-Cancer-Patient-to-Cancer-Survivor-Lost-in-Transition.aspx. Accessed September 2019

21 Hewitt M, Greenfield S, Stovall E, eds.; Institute of Medicine; National Research Council. From Cancer Patient to Cancer Survivor: Lost in Transition. Washington, D.C. : The National Academies Press; 2006

22 Levit LA, Balogh EP, Nass SJ, Ganz PA, eds.; IOM. Delivering HighQuality Cancer Care: Charting a New Course for a System in Crisis. Washington, D.C.: The National Academies Press; 2013

23 Birken SA, Deal AM, Mayer DK, Weiner BJ. Following through: the consistency of survivorship care plan use in United States cancer programs. JCancer Educ 2014;29(04):689-697

24 Ammon M, Arenja N, Leibundgut G, et al. Cardiovascular management of cancer patients with chemotherapy-associated left ventricular systolic dysfunction in real-world clinical practice. J Card Fail 2013;19(09):629-634

25 Clauser SB, Wagner EH, Aiello Bowles EJ, Tuzzio L, Greene SM. Improving modern cancer care through information technology. Am J Prev Med 2011;40(05, Suppl 2):S198-S207

26 Foraker RE, Shoben AB, Lopetegui MA, et al. Assessment of life's simple 7 in the primary care setting: The stroke prevention in healthcare delivery environments (sphere) study. Contemp Clin Trials 2014;38(02):182-189

27 Foraker RE, Kite B, Kelley MM, et al. Ehr-based visualization tool: adoption rates, satisfaction, and patient outcomes. EGEMS (Wash DC) 2015;3(02):1159

28 FDA. Digital health. 2018; Available at: https://www.fda.gov/MedicalDevices/DigitalHealth/default.htm. Accessed October 23, 2018

29 WHO. Classification of digital health interventions v1.0. 2018; Available at: http://apps.who.int/iris/bitstream/handle/10665/ 260480/WHO-RHR-18.06-eng.pdf;jsessionid=B42D38F5B9967374443366ED1CE122D0? sequence $=1$. Accessed October 23, 2018

30 Sharifi M, Adams WG, Winickoff JP, Guo J, Reid M, Boynton-Jarrett R. Enhancing the electronic health record to increase counseling and quit-line referral for parents who smoke. Acad Pediatr 2014; 14(05):478-484

31 Chrimes D, Kitos NR, Kushniruk A, Mann DM. Usability testing of avoiding diabetes thru action plan targeting (ADAPT) decision support for integrating care-based counseling of pre-diabetes in an electronic health record. Int J Med Inform 2014;83(09): 636-647

32 Zamora A, Fernández de Bobadilla F, Carrion C, et al; VALIDA Study Group; Network of Lipid Units of Catalonia (XULA). Pilot study to validate a computer-based clinical decision support system for dyslipidemia treatment (HTE-DLP). Atherosclerosis 2013;231 (02):401-404

33 Litvin CB, Ornstein SM, Wessell AM, Nemeth LS, Nietert PJ. Use of an electronic health record clinical decision support tool to improve antibiotic prescribing for acute respiratory infections: the ABX-TRIP study. J Gen Intern Med 2013;28(06):810-816

34 Razavi SA, Carter AB, Puskas JD, Gregg SR, Aziz IF, Buchman TG. Reduced red blood cell transfusion in cardiothoracic surgery after implementation of a novel clinical decision support tool. J Am Coll Surg 2014;219(05):1028-1036

35 Robbins GK, Lester W, Johnson KL, et al. Efficacy of a clinical decision-support system in an HIV practice: a randomized trial. Ann Intern Med 2012;157(11):757-766

36 Slev VN, Mistiaen P, Pasman HR, Verdonck-de Leeuw IM, van Uden-Kraan CF, Francke AL. Effects of eHealth for patients and informal caregivers confronted with cancer: A meta-review. Int J Med Inform 2016;87:54-67

37 Berry DL, Hong F, Halpenny B, et al. Electronic self-report assessment for cancer and self-care support: results of a multicenter randomized trial. JClin Oncol 2014;32(03):199-205

38 Jaatun EAA, Fallon M, Kofod-Petersen A, Halvorsen K, Haugen DF Users' perceptions on digital visualization of neuropathic cancerrelated pain. Health Informatics J 2019;25(03):683-700

39 Roberts AL, Fisher A, Smith L, Heinrich M, Potts HWW. Digital health behaviour change interventions targeting physical activity and diet in cancer survivors: a systematic review and metaanalysis. JCancer Surviv 2017;11(06):704-719

40 Belden J, Plaisant C, Johnson T, et al. Inspired EHRs: Designing for Clinicians. St. Louis, MO: The Curators of the University of Missouri; 2014

41 Nielsen J. The usability engineering lifecycle. In: Usability Engineering. San Diego, CA: Academic Press Inc.; 1993:71-114

42 Foraker RE, Shoben AB, Kelley MM, et al. Electronic health recordbased assessment of cardiovascular health: the stroke prevention in healthcare delivery environments (SPHERE) study. Prev Med Rep 2016;4:303-308

43 Lloyd-Jones DM, Hong Y, Labarthe D, et al; American Heart Association Strategic Planning Task Force and Statistics Committee. Defining and setting national goals for cardiovascular health promotion and disease reduction: the American Heart Association's strategic Impact Goal through 2020 and beyond. Circulation 2010;121(04):586-613

44 Shneiderman B, Plaisant C. Designing the user interface. 5th ed. Boston, MA: Pearson; 2010 
45 Li AC, Kannry JL, Kushniruk A, et al. Integrating usability testing and think-aloud protocol analysis with "near-live" clinical simulations in evaluating clinical decision support. Int J Med Inform 2012;81(11):761-772

46 Washington KT, Oliver DP, Gage LA, Albright DL, Demiris G. A multimethod analysis of shared decision-making in hospice interdisciplinary team meetings including family caregivers. Palliat Med 2016;30(03):270-278

47 Alpert JM, Krist AH, Aycock RA, Kreps GL. Applying multiple methods to comprehensively evaluate a patient portal's effectiveness to convey information to patients. J Med Internet Res 2016; 18(05):e112

48 Menon S, Smith MW, Sittig DF, et al. How context affects electronic health record-based test result follow-up: a mixed-methods evaluation. BMJ Open 2014;4(11):e005985

49 Bender JL, Yue RY, To MJ, Deacken L, Jadad AR. A lot of action, but not in the right direction: systematic review and content analysis of smartphone applications for the prevention, detection, and management of cancer. J Med Internet Res 2013;15(12):e287

50 Sandelowski M. Qualitative analysis: what it is and how to begin. Res Nurs Health 1995;18(04):371-375

51 Schwandt TA, Lincoln YS, Guba EG. Judging interpretations: but is it rigorous? Trustworthiness and authenticity in naturalistic evaluation. New Dir Eval 2007;2007(114):11-25

52 Guba E, Lincoln Y. Competing paradigms in qualitative research. In: Denzin NK, Lincoln YS, eds. Handbook of Qualitative Research. Thousand Oaks, CA: SAGE Publications, Inc.; 1994

53 Krippendorff K. Content Analysis: An introduction to Its Methodology. Thousand Oaks, CA: SAGE Publications, Inc.; 2004

54 Sittig DF, Singh $\mathrm{H}$. A new sociotechnical model for studying health information technology in complex adaptive healthcare systems. Qual Saf Health Care 2010;19(Suppl 3):i68-i74

55 Shay CM, Gooding HS, Murillo R, Foraker R. Understanding and improving cardiovascular health: An update on the american heart association's concept of cardiovascular health. Prog Cardiovasc Dis 2015;58(01):41-49

56 Sittig DF, Krall M, Kaalaas-Sittig J, Ash JS. Emotional aspects of computer-based provider order entry: a qualitative study. JAm Med Inform Assoc 2005;12(05):561-567

57 Henriksen K, Joseph A, Zayas-Cabán T. The human factors of home health care: a conceptual model for examining safety and quality concerns. J Patient Saf 2009;5(04):229-236

58 NSAI. Ergonomic Human-System Interaction-Part 11: Usability: Definitions and Concepts. 2nd ed. Geneva, Switzerland: European Committee for Standardization; 2018

59 West P, Van Kleek M, Giordano R, Weal M, Shadbolt N. Information quality challenges of patient-generated data in clinical practice. Front Public Health 2017;5:284

60 Cheung WY, Neville BA, Cameron DB, Cook EF, Earle CC. Comparisons of patient and physician expectations for cancer survivorship care. JClin Oncol 2009;27(15):2489-2495

61 Cheung WY, Aziz N, Noone AM, et al. Physician preferences and attitudes regarding different models of cancer survivorship care: a comparison of primary care providers and oncologists. J Cancer Surviv 2013;7(03):343-354

62 Blanch-Hartigan D, Forsythe LP, Alfano CM, et al. Provision and discussion of survivorship care plans among cancer survivors: results of a nationally representative survey of oncologists and primary care physicians. JClin Oncol 2014;32(15):1578-1585

63 Meiklejohn JA, Mimery A, Martin JH, et al. The role of the GP in follow-up cancer care: a systematic literature review. JCancer Surviv 2016;10(06):990-1011
64 Singer S, Gianinazzi ME, Hohn A, Kuehni CE, Michel G. General practitioner involvement in follow-up of childhood cancer survivors: a systematic review. Pediatr Blood Cancer 2013;60(10): 1565-1573

65 Berry DL, Nayak MM, Abrahm JL, Braun I, Rabin MS, Cooley ME. Clinician perspectives on symptom and quality of life experiences of patients during cancer therapies: Implications for eHealth. Psychooncology 2017;26(08):1113-1119

66 Puszkiewicz P, Roberts AL, Smith L, Wardle J, Fisher A. Assessment of cancer survivors' experiences of using a publicly available physical activity mobile application. JMIR Cancer 2016;2 (01):e7

67 Robertson MC, Tsai E, Lyons EJ, et al. Mobile health physical activity intervention preferences in cancer survivors: A qualitative study. JMIR Mhealth Uhealth 2017;5(01):e3

68 Pugh G, Hough RE, Gravestock HL, Jackson SE, Fisher A. The health behavior information needs and preferences of teenage and young adult cancer survivors. JAdolesc Young Adult Oncol 2017;6(02):318-326

69 Smith SK, Kuhn E, O'Donnell J, et al. Cancer distress coach: Pilot study of a mobile app for managing posttraumatic stress. Psychooncology 2018;27(01):350-353

70 McCarroll ML, Armbruster S, Pohle-Krauza RJ, et al. Feasibility of a lifestyle intervention for overweight/obese endometrial and breast cancer survivors using an interactive mobile application. Gynecol Oncol 2015;137(03):508-515

71 Henriksen PA. Anthracycline cardiotoxicity: an update on mechanisms, monitoring and prevention. Heart 2018;104(12): 971-977

72 Leong SL, Chaiyakunapruk N, Tassaneeyakul W, Arunmanakul P, Nathisuwan S, Lee SWH. Roles of pharmacogenomics in nonanthracycline antineoplastic-induced cardiovascular toxicities: A systematic review and meta-analysis of genotypes effect. Int J Cardiol 2019;280:190-197

73 Darby SC, Ewertz M, McGale P, et al. Risk of ischemic heart disease in women after radiotherapy for breast cancer. N Engl J Med 2013; 368(11):987-998

74 Armenian SH, Lacchetti C, Barac A, et al. Prevention and monitoring of cardiac dysfunction in survivors of adult cancers: American society of clinical oncology clinical practice guideline. J Clin Oncol 2017;35(08):893-911

75 Zamorano JL, Lancellotti P, Rodriguez Muñoz D, et al; ESC Scientific Document Group. 2016 ESC Position Paper on cancer treatments and cardiovascular toxicity developed under the auspices of the ESC Committee for Practice Guidelines: The Task Force for cancer treatments and cardiovascular toxicity of the European Society of Cardiology (ESC). Eur Heart J 2016;37 (36):2768-2801

76 Ammenwerth E, Mansmann U, Iller C, Eichstädter R. Factors affecting and affected by user acceptance of computer-based nursing documentation: results of a two-year study. JAm Med Inform Assoc 2003;10(01):69-84

77 Hersh W. Health care information technology: progress and barriers. JAMA 2004;292(18):2273-2274

78 Shanahan CW, Sorensen-Alawad A, Carney BL, et al. The implementation of an integrated information system for substance use screening in general medical settings. Appl Clin Inform 2014;5 (04):878-894

79 Kirkovits T, Schinkoethe T, Drewes C, et al. Ehealth in modern patient-caregiver communication: High rate of acceptance among physicians for additional support of breast cancer patients during long-term therapy. JMIR Cancer 2016;2(02):e14 